\title{
Ameloblastoma plexiforme del maxilar: manejo quirúrgico y protético. A propósito de un caso
}

\section{Carini F*, Riazzoli $\mathrm{G}^{* *}$, Vignoletti $\mathrm{F}^{* * *}$ Baldoni $\mathrm{M}^{* * * *}$}

\section{RESUMEN}

El ameloblastoma es un tumor odontógeno benigno de derivación epitelial, de elevada invasión local, crecimiento intermitente y con alta probabilidad de recidiva.

Puede dividirse en los siguientes tipos histológicos: folicular, plexiforme, acantomatoso, a células basales y granulares.

El ameloblastoma folicular es el subtipo histológico más común.

Se manifiesta prevalentemente durante la tercera, cuarta y quinta década de vida, sin predilección para el sexo, aun así se puede encontrar en cualquier grupo de edad incluyendo niños.

La mayoría de los ameloblastomas se encuentra sobretodo en mandíbula (al nivel del ángulo y rama).

Las características clínicas, incluso si complementadas con radiografías y muestras histológicas, no son útiles a la hora de predeterminar el comportamiento biológico y por lo tanto el pronóstico de un ameloblastoma individual.

Los autores presentan un caso localizado de ameloblastoma plexiforme localizado en la parte posterior del maxilar de un hombre de 30 años; el término "plexiforme" se refiere al aspecto de anastomosis de los islotes del epitelio odontógeno, en contraste con el tipo «folicular».

En este artículo se discuten importantes aspectos de la patología, del tratamiento quirúrgico y protésico.

En conclusión, es necesaria una cooperación estricta a largo plazo de un grupo de clínicos, patólogos, cirujanos y prostodoncistas, para ofrecer el mejor tratamiento individualizado en cada caso.

Palabras clave: Ameloblastoma plexiforme, cavidad oral, tumor odontógeno.

\section{SUMMARY}

The ameloblastoma is a clinically persistent benign tumor derived from odontogenic epithelium, locally invasive, intermittent in growth and with high a probability of recurrence.

The following histologic patterns may be distinguished: follicular, plexiform, spindle cell, acanthomatous, basal cell type and granular cell.

The follicular ameloblastoma is the most common histologic tipe.

The majority of patients are in 3th, 4th and 5th decade, but can be found in any age group including children. The majority of ameloblastomas are observed in the mandible (mostly angle or ramus).

Clinical features, even if combined with radiology or histology findings, are not useful when trying to determine the biological behaviour and therefore the prognosis of an individual ameloblastoma.

The authors report a case of plexiform ameloblastoma in the posterior maxilla, in male 30 year old otherwise healthy; the term "plexiform" refers to the appearance of anastomosing islands of odontogenic epithelium in contrast to a follicular pattern.

Important questions on pathology, surgical and prosthetic therapy are discussed in this paper.

In conclusion, a close cooperation of clinicians, pathologists, surgeons and prosthesists is necessary and very important over a long period of time for the best management of each individual case. 
Key words: Plexiform ameloblastoma, oral cavity, odontogenic tumor.

Fecha de recepción: Junio 2006.

Aceptado para publicación: Octubre 2006.

* Investigador Universitá degli Studi di Milano-Bicocca. Departamento de Odontoestomatología.

** Universitá degli Studi di Milano-Bicocca.

*** Master de Periodoncia Universidad Complutense de Madrid.

***** Profesor ordinario Universitá degli Studi di Milano-Bicocca.

Carini F, Riazzoli G, Vignoletti F, Baldoni M. Ameloblastoma plexiforme del maxilar: manejo quirúrgico y protético. A propósito de un caso. Av. Odontoestomatol 2007; 23 (1): 11-20.

\section{INTRODUCCIÓN}

Epidemiológicamente el ameloblastoma representa el $11 \%$ de los tumores odontógenos aproximadamente, y el $0.14 \%$ de todas las neoplasias. Algunos estudios (1.2) definen el ameloblastoma como un tumor muy raro de mandíbula y maxilar, con una incidencia particularmente elevada en África del este (3).

Se manifiesta prevalentemente durante la tercera, cuarta y quinta década de vida, sin predilección por el sexo (4), con la mayoría de los pacientes entre los 40 y 50 años.

Sin embargo la lesión se puede encontrar en cualquier grupo de edad, afectando hasta los niños $(5,6)$.

Se localiza con más frecuencia a nivel de la mandíbula con respecto al maxilar: el ángulo y la rama mandibulares junto al área de los terceros molares inferiores representan las localizaciones más afectadas $(1,7)$.

Los ameloblastomas periféricos son muy raros (8): son tumores de tejidos blandos tanto primarios como secundarios; estos últimos aparecen después de la cirugía.

Los ameloblastomas (sinónimos: adamantinoma, adamantoblastoma, quistes multiloculares, son neoplasias odontógenas benignas de agresividad local elevada y probabilidad del recidiva.

Tienen origen en el epitelio germinativo de los elementos dentales de maxilar y mandíbula.
El origen potencial del tipo epitelial incluyen el órgano del esmalte, el epitelio reducido del esmalte, los restos epiteliales de Serres o de Malassez y el epitelio de los quistes odontógenos (sobre todo los quistes dentígeros); todavía permanece actualmente desconocido el estímulo a la transformación neoplásica de las células epiteliales.

La descripción original del tumor fue de Cassock en 1827, Broca en 1868, presentó el primer caso en literatura científica y Falkson en 1879 realizó la primera descripción histológica completa.

El término ameloblastoma fue propuesto por Churchill en 1934 (9) a substitución del anterior término adamantinoma, introducido por Malassez en 1885 indicando erróneamente la formación de tejidos duros.

Se han descrito numerosas variedades histológicas de ameloblastoma. Algunas demuestran un solo subtipo histológico; en diferentes cuadros histológicos otras se asocian dentro de una misma lesión.

La característica común a casi todos los subtipos es la polarización de las células que rodean los islotes de proliferación, de una manera similar a cuánto sucede en el ameloblastoma del órgano del esmalte.

El área más central de la lesión recuerda el aspecto del retículo estrellado del órgano del esmalte.

La otra particularidad es la proliferación de las células tumorales en los islotes neoplásicos, que es similar al proceso de neoformación dentaria. 
Considerando que el curso histológico de estas lesiones varía, se pueden diferenciar distintos subtipos $(2,10)$ : el más común es el folicular; ocasionalmente, las células neoplásicas proliferan en el contexto del epitelio, dando origen al ameloblastoma plexiforme.

Los ameloblastomas se definen acantomatosos o fusiformes si el área central de los folículos se constituye por células escamosas o alargadas y de células basales si poseen las características histológicas similares al carcinoma de células basales cutáneo.

Finalmente el subtipo de células granulares posee una granulación citoplásmica de las células centrales del folículo.

El ameloblastoma plexiforme en su variante uniquística presenta epitelio ameloblástico en el contexto de la pared quística. En algunos casos raros, la involución terminal representa la degeneración maligna.

Los raros ameloblastomas malignos se manifiestan principalmente en sujetos jóvenes con una localización mandibular más frecuente que maxilar. Estos tumores producen metástasis hacia los linfonodos regionales o a órganos distantes.

Las metástasis se localizan, en orden de frecuencia, en pulmón, linfonodos regionales, pleura, vértebras, huesos del cráneo, diafragma, hígado y parótida y, más ocasionalmente, bazo y riñones (11).

Las lesiones malignas se han subdividido en dos subtipos: ameloblastoma maligno y el carcinoma ameloblástico. El primero se caracteriza por lesiones primitivas y metastásicas histológicamente bien diferenciadas, mientras que el segundo presenta lesiones con un grado de menor diferenciación y de atipia citológica y de figuras mitóticas anormales.

Considerando la edad del paciente, localización y aspectos radiográfícos de la lesión, el diagnóstico diferencial se limita substancialmente a tres grupos de lesiones de los maxilares:

1. Tumores odontógenos: variedad radiotransparente del tumor odontógeno epitelial calcificante (CEOT) y los mixomas.
2. Quistes: quiste dentígero y queratoquiste odontógeno.

3. Lesiones odontógenas benignas: granuloma de células gigantes centrales, el fibroma osificante, hemangioma central y la histiocitosis idiopática, radiográficamente similares al ameloblastoma en los pacientes relativamente jóvenes.

Histológicamente algunos ameloblastomas, especialmente los plexiformes uniquísticos y poliquísticos, pueden parecer a los quistes odontógenos por la presencia de hiperplasia de las células parietales; caracterizándose por células basales dispuestas en palizada y por escasas células flogísticas.

A veces los ameloblastomas del maxilar aparecen poco diferenciados y deben diferenciarse del adenocarcinoma y de los carcinomas a las células escamosas del seno maxilar.

\section{CASO CLÍNICO}

En el mes de Marzo de 2005, un paciente de 30 años se presenta a observación en el departamento de Cirugía Odontoestomatológica de la Università degli studi di Milano-Bicocca, en el Hospital San Gerardo di Monza, por una consulta sobre el elemento dental 2.8 (tercer molar superior izquierdo). El paciente no presentaba sintomatología clínica relevante, excepto por un dolor leve, divulgado en la zona del tercer molar.

Al examen extraoral no se evidenciaban signos relevantes y la palpación del linfonodos del cuello demostraba una situación de normalidad.

El examen intraoral revelaba la inclusión parcial mucosa del elemento 2.8, sin variaciones particulares de color y de consistencia a la palpación de la zona bajo examen.

El análisis radiográfico con ortopantomografía (fig. 1), el elemento 2.8 presentaba zonas de radiotransparente aumentadas.

El paciente, que carecía de patologías sistémicas, en el mes de Marzo de 2005 recibe la extracción del elemento 2.8 y biopsia de los tejidos adyacentes, de 


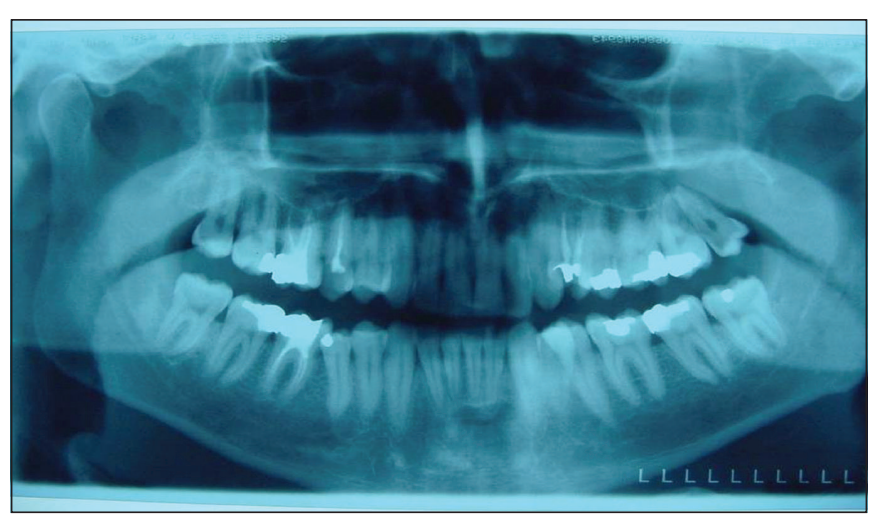

Fig. 1. Ortopantomografía inicial.

las dimensiones de aproximadamente $1.0 \times 1.0 \times 1.0$ centímetros.

El diagnóstico en base al informe histológico era de ameloblastoma plexiforme (fig. 2).

Al profundizar el diagnóstico radiográfico a través de la tomografía axial computarizada con programa Dentascan (fig. 3), demuestra la afectación de una amplia zona del maxilar izquierdo que alcanzaba la base de la órbita.

En el mes de mayo 2005 bajo anestesia general se realiza una hemi-maxillectomia con amplios márgenes de seguridad a partir del primer premolar supe-

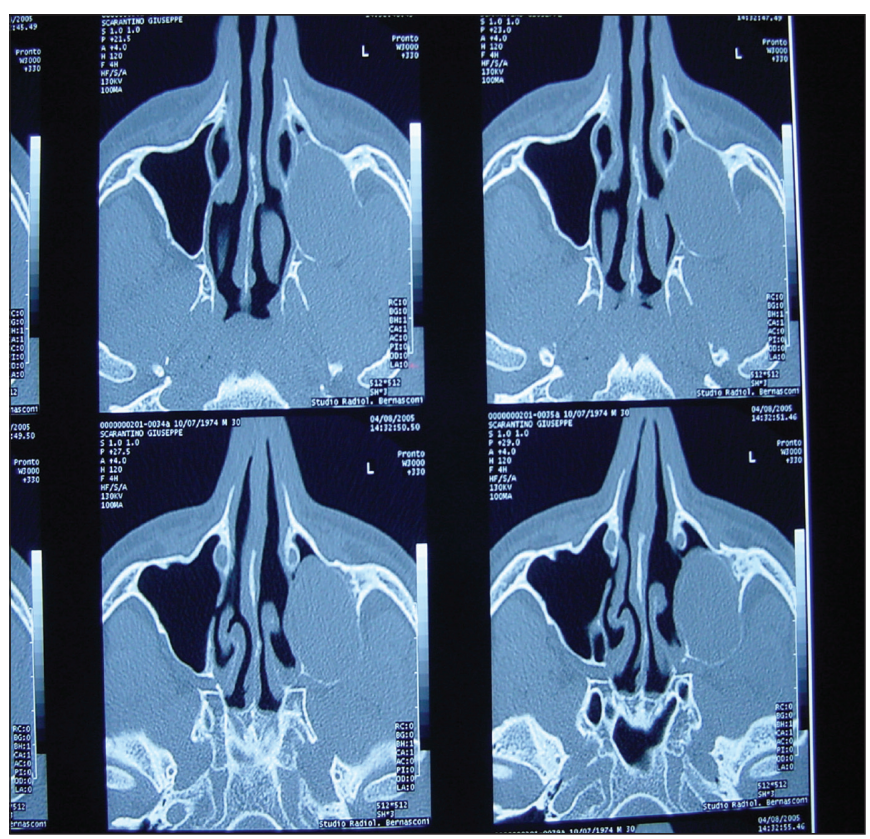

Fig. 3. TAC maxilar.

rior izquierdo (figs. 4 y 5), según el principio, apoyado por muchos autores, que inspira la terapia de los tumores malignos, de "resecar en tejidos aparentemente sanos".

Se hizo un ulterior análisis histológico del espécimen quirúrgico que confirmó el diagnóstico de ameloblastoma plexiforme.

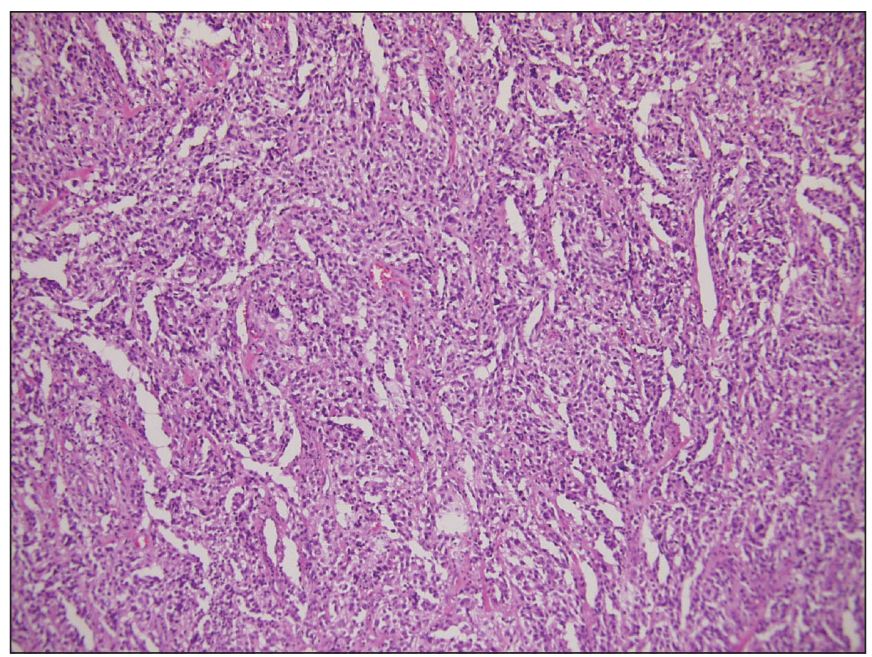

Fig. 2. Examen histológico.

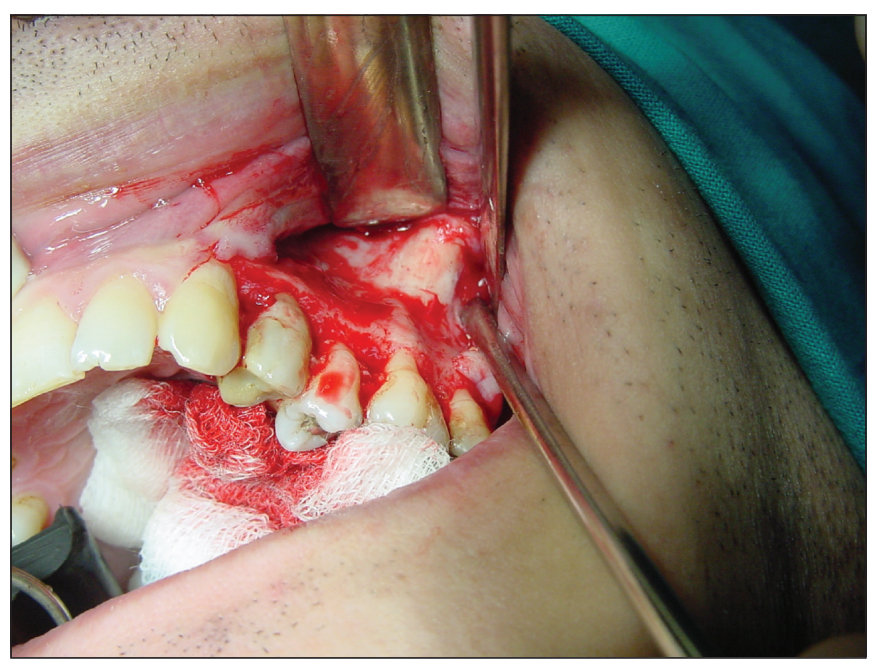

Fig. 4. Fase inicial de la intervención. 


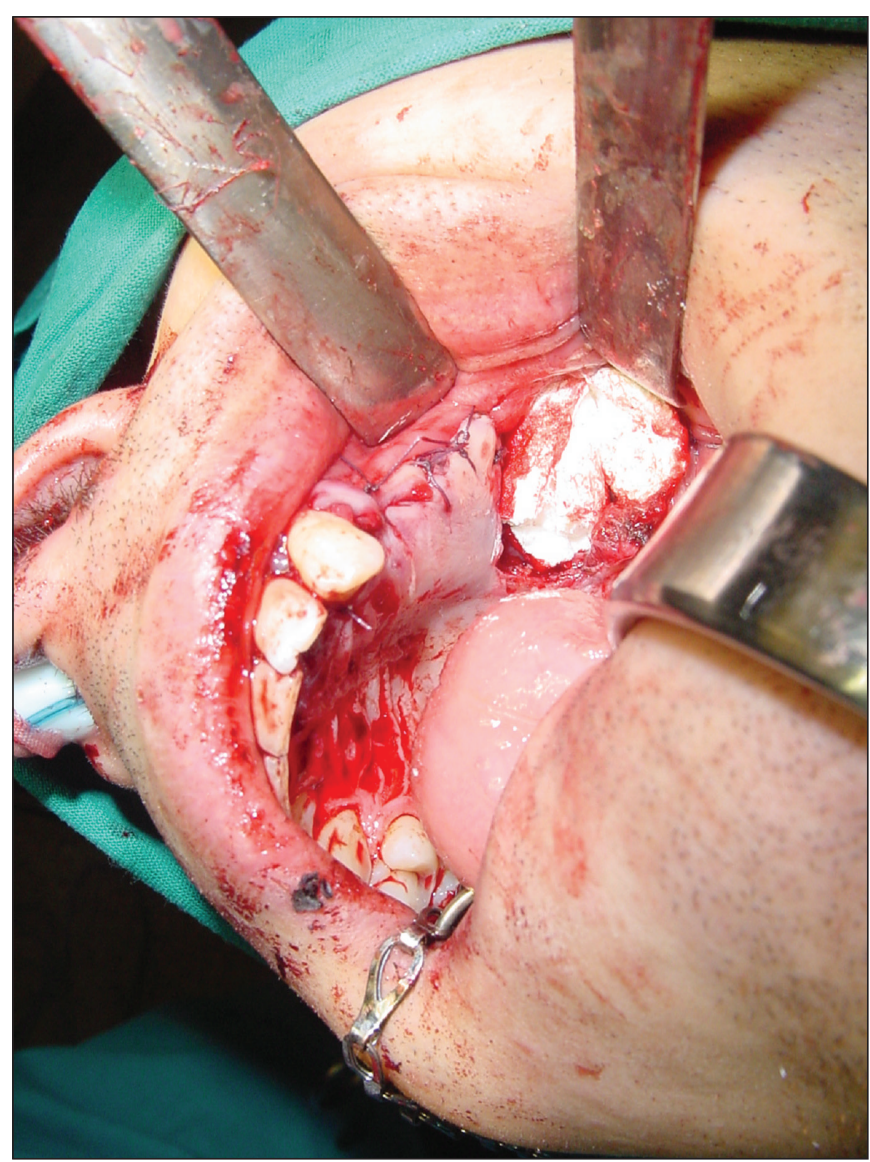

Fig. 5. Fase final de la intervención.

Con respecto al tratamiento porotésico, antes de la cirugía se realizaron impresiones de los maxilares y tras el colado se realizó una simulación de la intervención quirúrgica en los modelos de escayola.

Con el auxilio del TAC se establecieron los márgenes de la resección, con particular atención al margen anterior, importante para no perjudicar el correcto posicionamiento de la prótesis palatina.

La prótesis de obturación palatina era mucosoportada y dento-retenida con ganchos en los elementos 2.3 y controlateralmente 1.4 y 1.5 y 1.7 y 1.8. (fig. 7). A intervención terminada se averiguó la adaptación del manufacto protésico que resultó adecuada (fig. 8).

La figura 9 demuestra cómo a los 6 meses en correspondencia de la zona de resección se observan tejidos normotróficos y bien vascularizados.

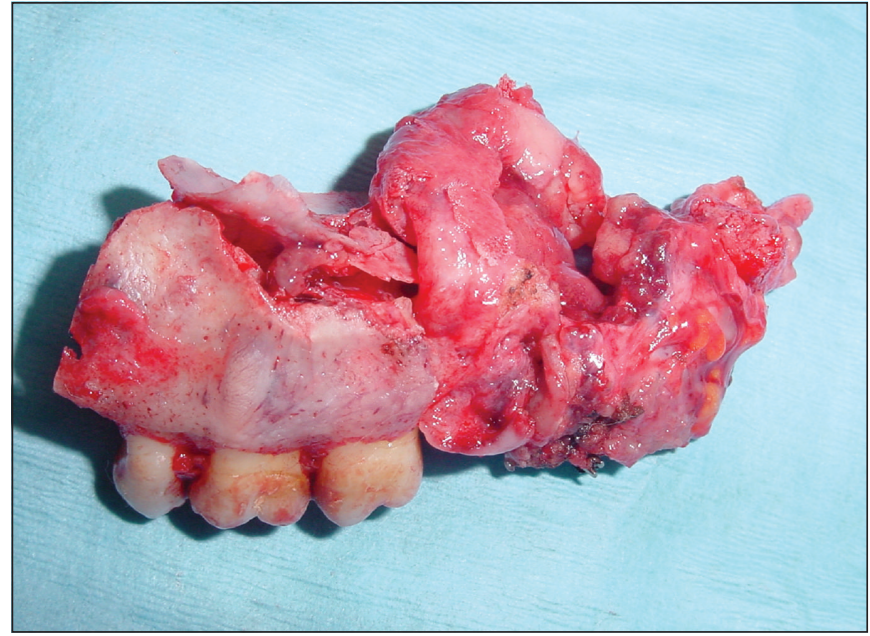

Fig. 6. Bloque asportado.

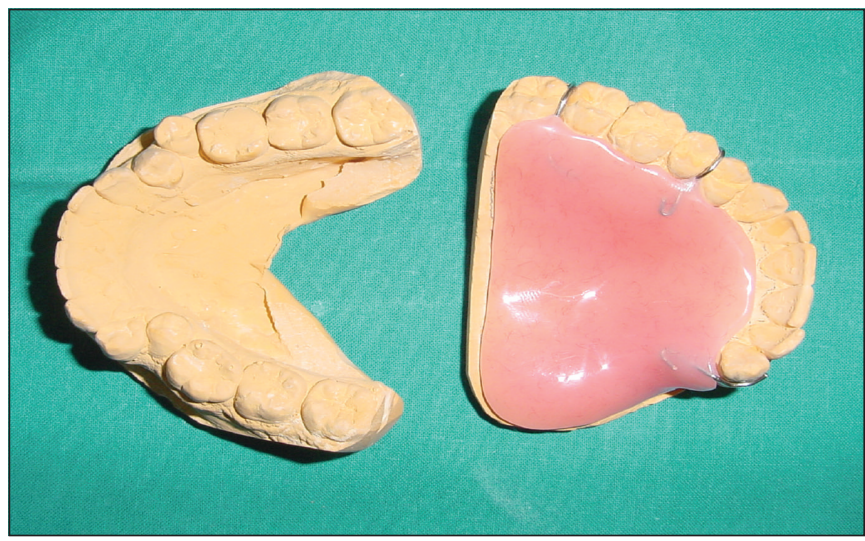

Fig. 7. Prótesis.

Se ha controlado y adaptado periódicamente la prótesis a las condiciones de los tejidos según la cicatrización y se estimará la posibilidad de añadir los dientes, manteniéndolos fuera de oclusión, hasta la fase de reconstrucción con implantes.

\section{DISCUSIÓN}

El ameloblastoma es normalmente asintomático en sus fases iniciales y a menuda se observa ocasionalmente durante una exploración radiográfica de rutina. En las fases mas avanzadas aparece como una lesión de lento crecimiento capaz de determinar una progresiva tumefacción del segmento óseo interesado. 


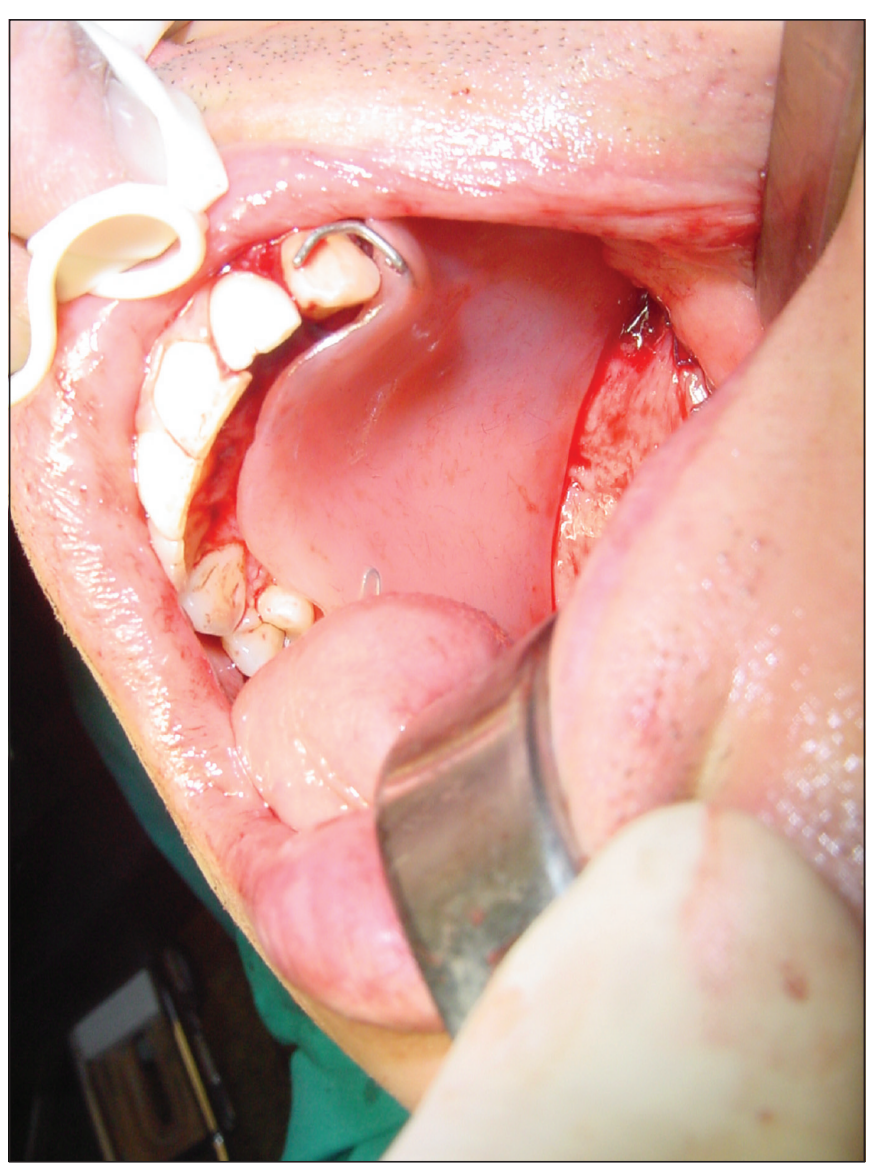

Fig. 8. Prótesis in situ.

Con respecto al comportamiento biológico, se trata de un tumor benigno con agresividad local, capaz de infiltrar las trabéculas óseas.

Se pueden observar movilidad de los elementos dentales en la zona implicada, maloclusión, perforación de las mucosas y obstrucción nasal en el caso de localización maxilar.

La actitud terapéutica de este tipo de neoplasia es su propensión característica a la recidiva (también frente a una actitud quirúrgica correcta), que surge de la posibilidad de dejar lesiones microscópicas periféricas de la eventualidad de un origen pluricéntrico del tumor.

El aspecto de la tendencia a la recidiva de este tumor se ha examinado particularmente por Smith (12), que ha encontrado en 40 de los casos tratados, 7 casos de recidiva. Esta proporción, que no es parti-

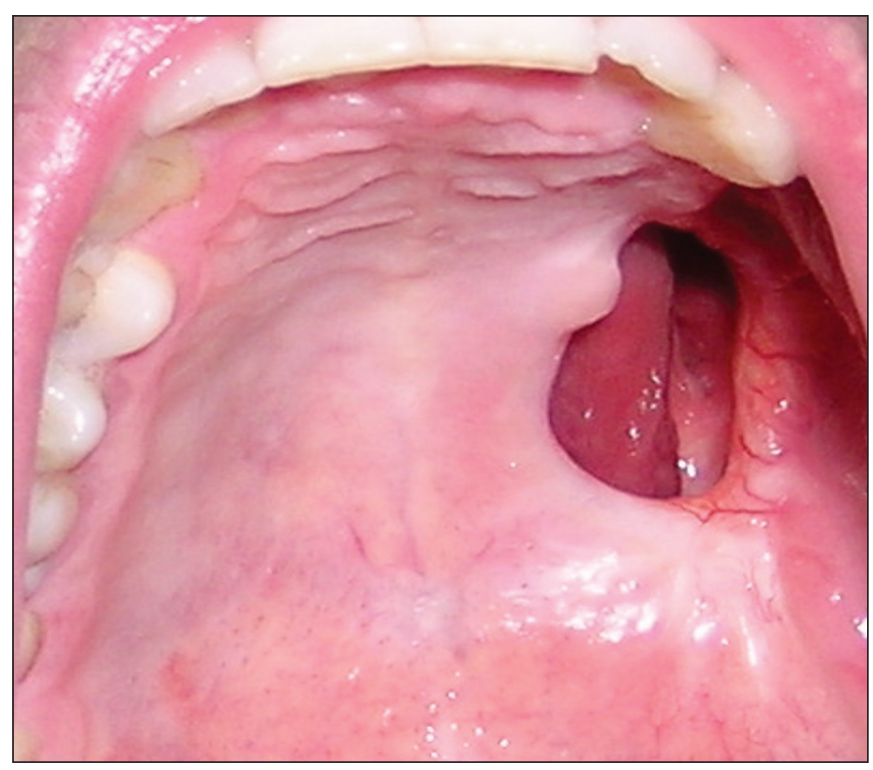

Fig. 9. Cicatrización a 6 meses.

cularmente alarmante, es el periodo marcado de latencia descrita también por otros autores que tiene que alarmar: de un mínimo de 4 años (1 caso), a un medio de 10 años (5 casos), hasta los 20 años en los restantes casos.

La enucleación simple del tumor, se podría tomar en consideración en los casos raros en que el hueso circundante presente una buena reactividad y la conservación de su trabeculado.

Otro criterio que se considera en la valoración clínica difícil de la agresividad del ameloblastoma, es su comportamiento con respecto a las raíces dentales contiguas; una erosión de los tejidos radiculares indica mas severidad, mientras que el movimiento simple de los dientes que se percibe a través de movilidad, es menos preocupante.

El examen diagnóstico estándar es la radiografía panorámica, mientras que una tomografía está indicada en la fase de planificación quirúrgica; puede aparecer como un área radiotransparente más o menos extensa, con los caracteres de una lesión unilocular muy delimitada y con un aspecto no distinguible de una lesión quística, y también puede aparecer bajo forma de las lesiones poliquísticas, compartimentadas por sectores óseos muy definidos en pa- 
nal de abejas. En este último caso el examen radiográfico es muy demostrativo.

La relación con un elemento dental incluido, implica un diagnóstico diferencial con los quistes foliculares.

En esta tipología de tumores las clasificaciones histológicas tienen gran importancia, aunque no existan correlaciones seguras entre los subtipos y el comportamiento clínico.

Muchos aspectos vuelven a entrar en las valoraciones del plan del tratamiento, del pronóstico y de los mantenimientos periódicos.

En tal sentido se ha propuesto una subdivisión en dos grupos clínico-histológicos en relación a las modalidades de tratamiento y al pronóstico (13):

1. Sólidos o multifocales.

2. Quísticos (uniquístico).

Los primeros están asociados a un mayor número de recidivas (de 50 a 90\%) y a una mayor agresividad, exigiendo un tratamiento más agresivo.

Con el intento de reducir el número de subtipos, se ha propuesto una diferenciación clínica del ameloblastoma, en sólidos o poliquísticos, uniquísticos y periféricos (14).

El plan del tratamiento puede diferenciarse en función de las localizaciones y del aspecto radiográfico: para las localizaciones mandibulares, en caso de tumores uniloculares con márgenes netos y no particularmente extendidos (con corticales integras) y para los ameloblastomas periféricos, el tratamiento puede ser de tipo conservador, con enucleación que se asocia a un curetaje enérgico de las paredes.

En caso de recidiva o de tumor poliquístico, con erosión de las corticales, se indica un tratamiento más agresivo que consiste en la resección amplia con márgenes de seguridad amplios (por lo menos 1 centímetro más allá de los límites radiológicos).

El mantenimiento de la continuidad del margen inferior de la mandíbula, simplifica la reconstrucción y la obtención de un buen resultado estético.
Otros autores atribuyen un valor pronóstico tanto a las características radiográficas como histológicas, y a la edad (15).

Los ameloblastomas maxilares se consideran más agresivos y con el pronóstico peor, mientras que los uniquísticos se consideran menos agresivos (16).

De una manera generalizada, ante la duda, el tratamiento mejor es sin duda una amplia exéresis de la lesión.

Para las localizaciones maxilares, sobretodo inherentes los sectores latero-posteriores como en el caso presentado, se indican de entrada cirugías más agresivas, al considerar la capacidad de desarrollarse de forma asintomática y poder infiltrar el seno maxilar, la fosa pterigopalatina, la fosa temporal y la base del cráneo (17).

La frecuencia de recidiva tras tratamiento conservador va del $50 \%$ hasta el $90 \%$ (18).

En la revisión de la literatura se pueden encontrar varios conceptos de terapia del ameloblastoma, más o menos específicos para cada subtipo.

Pinsolle J. y cols. (19) recomienda la resección segmentaria como tratamiento elegido para la mandíbula, y la resección marginal solamente para los tumores primarios de dimensiones pequeñas.

Olaitan y Adekeye (16), como Gardner y Corio (20) por ejemplo, afirman que es suficiente el curetaje para el tratamiento del ameloblastoma plexiforme del uniquístico a condición de que el tumor no haya invadido el tejido conectivo periférico.

Curi y cols. (21) han sugerido que el tratamiento de los ameloblastomas sólidos o multiquísticos de la mandíbula con curetaje seguido por criocirugía con spray de nitrógeno líquido, puede reducir el porcentaje del recidiva local y disminuir la indicación a la resección radical.

La radioterapia en el tratamiento de los ameloblastomas, considerados tumores radiorresistententes, tiene un papel muy discutido pero sustancialmente limitado a los casos en los cuales la cirugía sería excesivamente destructiva. 
El alcance de la intervención resectivo no consiste solamente en la eliminación completa de la lesión, erradicando las células neoplásicas que podrían proliferar y provocar una recidiva, sino que también en la optimización funcional: los procedimientos reconstructivos, por tanto, se deben planear antes de la cirugía y el objetivo determina la elección entre las diferentes técnicas a la disposición.

Los métodos a disposición varían mucho en función de la naturaleza de la lesión: la identificación histológica de la lesión es, sin embargo, un elemento inevitable en la elaboración del plan del tratamiento.

Los factores a considerar en la decisión entre los distintos abordajes quirúrgicos son numerosos:

- Agresividad de la lesión: el diagnóstico histológico identifica y dirige el tratamiento puesto que, más que otros puede condicionar el pronóstico.

- Localización anatómica de la lesión: la posición de la lesión puede complicar la escisión quirúrgica, e influenciar negativamente el pronóstico.

- Proximidad de lesiones a los dientes y a las estructuras neurovasculares: el intento de preservar estas estructuras puede minar la radicalidad de la cirugía.

- Dimensiones del tumor.

- Duración de la lesión: las lesiones de lento crecimiento parecen tener un pronóstico postquirúrgico mejor.

Simplificando, podemos decir que el tratamiento conservador esté limitado al ameloblastoma mandibular de aspecto uniquístico y de pequeñas dimensiones, especialmente con localización mandibular, respecto de las corticales y también los periféricos.

El tratamiento agresivo, es decir una remoción con amplios márgenes de tejido sano, se indica para el ameloblastoma solido-multiquístico o a localización maxilar.

El tratamiento radical se indica para raros ameloblastomas malignos que sobretodo afectan a sujetos jóvenes (tercera década de edad) y para el carcinoma ameloblástico; en el tratamiento de las lesiones malignas, es necesario poner la atención también en otras problemáticas como la presencia y el grado de la metástasis, la radiosensibilidad y la quimiosensi- blidad del tumor, la edad y las condiciones físicas generales del paciente y de sus expectativas.

Visto el alto porcentaje de recidivas, los pacientes deben entrar en un programa de control de al menos 5 años, pero recomendamos controles clínicos y radiográficos a largo plazo.

Con respecto a la rehabilitación protésica y funcional de estos pacientes que han recibido cirugías resectivas, evidenciamos como resulte menos predecible y más compleja, con respecto a rehabilitaciones en pacientes no oncológicos con similares defectos.

Los límites de la rehabilitación dental son imputables a la distorsión de la anatomía oral, a la presencia de tejido cicatricial, a la modificación de la topografía ósea y a la perdida del vestíbulo.

La valoración prequirúrgica del prostodoncista desempeña un papel fundamental: la toma de impresiones y el estudio de modelos permite al cirujano establecer los márgenes de la resección.

Es necesario preservar la mayor cantidad de hueso maxilar posible: hasta un mínimo soporte óseo puede aumentar enormemente la estabilidad de la prótesis.

El pronóstico de este tipo de rehabilitación está relacionado estrictamente a la dimensión del defecto y su localización.

En nuestro caso, el anclaje a los dientes residuales, ha permitido ofrecer al paciente un discreto grado de retención protésica.

La necesidad de reconstruir la porción ósea de un maxilar es generalmente menos crucial con respecto a una reconstrucción de la mandíbula: el uso de un obturador ha resultado suficiente para reestablecer un contorno facial armónico y una funcionalidad aceptable, cerrando la comunicación oronasal.

Kornblith y a. (22) han estudiado la adaptación psicosocial de los pacientes tratados con resección maxilar por diagnóstico de tumor maligno del paladar duro o del seno maxilar, rehabilitados por medio de obturadores para el restablecimiento de la fonética y 
alimentación, obteniendo una mejoría de la calidad de vida que se reflejaba en una menor dificultad en la pronunciación de las palabras, masticación y al tragar alimentos y además de válido y aceptable un aspecto estético.

Los principios básicos en la realización de la prótesis son los mismos a los que nos referimos en el tratamiento del desdentado parcial o total (23); se podría considerar la posibilidad de aligerar y mejorar su estabilidad.

\section{CONCLUSIONES}

En este tipo de neoplasia, no existe un tratamiento estandarizado y cada caso tendría que analizarse por separado.

No estamos por esto autorizados a ignorar las numerosas observaciones e indicaciones recientemente publicadas en la literatura científica $(24,25)$.

Resulta indispensable un buen encuadre nosológico de la neoplasia, una programación terapéutica cuanto más escrupulosa y que tenga en cuenta también las expectativas del paciente.

Es necesaria por tanto una colaboración válida entre patólogos, cirujanos y prostodoncistas para proveer el mejor tratamiento personalizado de cada caso y recomendar controles postoperatorios clínicos y radiográficos a largo plazo. No es siempre fácil mediar entre una actitud terapéutica de tipo conservador o radical, pero la opción debe siempre dirigirse principalmente a la tutela la salud del paciente, no solamente a corto y medio plazo, sino que también a largo plazo.

En espera de una cirugía de reconstrucción con implante, el obturador ofrece un adecuado nivel estético, funcional, y permite satisfacer el lado psicológico del paciente mejorando su calidad de vida.

\section{BIBLIOGRAFIA}

1. ME Donath K. Odontogene Tumoren. In Steinhäuser EW ed. Fortschritte KieferGesichtschirurgie. Stuttgart: Thieme. 1986; 86-91.
2. Mosqueda-Taylor A, Ledesma-Montes C, Caballero-San-doval S, Portilla-Robertson J, Ruiz-Godoy Rivera LM, Meneses-Garcia A. Odontogenic tumors in Mexico: a collaborative retrospective study of 349 cases. Oral Surg Oral Med Oral Pathol Oral Radiol Endod. 1997; 84: 672-5.

3. Small IA, Waldron CA. Ameloblastoma of the jaws. Oral Surg 1955; 8: 281-97.

4. Brusati R, Chiapasco M. Elementi di chirurgia oro-maxillo-facciale. Masson, Milano, 1999.

5. Bingham HG, Boyle PO. Abbreviated case report. Mandibular ameloblastoma in a 3 year old boy. Oral Surg Oral Med Oral Pathol 1971; 32: 7580.

6. Langer E. Histopathologie der Tumoren der Kiefer- und der Mundhöhle. Stuttgart: Thieme. 1958; 154.

7. Ueno S, Nakamura S, Mushimoto K, Shirasu R. A clinic pathologic study of ameloblastoma. J Oral Maxillofac Surg. 1986; 44: 361-5.

8. Gurol M, Burkes EJ. Peripheral ameloblastoma. J Periodontol. 1995; 66: 1065-8.

9. Churchill HR. Histologic differentiation between certain dentigerous cysts and ameloblastoma. Dent. Cosmos. 1934; 76:1173-8.

10. Prein J, Remagen W, Spiessl B, Uehlinger E : eds. Atlas der Tumoren des Gesichtsschädels. Berlin: Springer. 1985; 82.

11. Inoue $\mathrm{N}$, Shimojiyo $M$, Iwai $\mathrm{H}$ et al.: Malignant ameloblastoma with pulmonary metastasis and hypercalcemia. Report of an autopsy case and review of the literature AJCP. 1988; 90; 474-81.

12. Smith I. Recurrent ameloblastoma of the mandible. J. Max Fac. Surg. 1976; 4: 1-7.

13. Regezi J.A, Sciubba J.J. : Oral Pathology-Clinical pathologic correlations, W.B. Saunders Company, Philadelphia, Pennsylvania. 1999; $3^{\text {rd }}$ Edition. 
14. Gardner DG. Some current concepts on the pathology of ameloblastomas. Oral Surg Oral Med Oral Pathol Oral Radiol Endod. 1996; 82: 660-9.

15. Ueno S, Mushimoto K, Shirasu R. Prognostic evaluation of ameloblastoma based on histologic and radiographic typing. J. Oral Maxillofac. Surg. 1989; 47: 11-5.

16. Olaitan AA, Adekeye EO. Unicystic ameloblastoma of the mandible: a long-term follow-up. J Oral Maxillofac Surg. 1997; 55: 345-8.

17. Komisar A. Plexiform ameloblastoma of the maxilla with extension to the skull base. Head $\mathcal{E}$ Neck Surgery. 1984; 7: 172-5.

18. Gardner DG, Pecak AMJ. The treatment of ameloblastoma based on pathologic and anatomic principles. Cancer. 1980; 46: 2514-9.

19. Pinsolle J, Michelet V, Coustal B, Siberchicot F, Michelet FX. Treatment of ameloblastoma of the jaws. Arch Otolaryngol Head Neck Surg. 1995; 121: 994-6.

20. Gardner DG, Corio RL. The relationship of plexiform unicystic ameloblastoma to conventional ameloblastoma. Oral Surg Oral Med Oral Pathol 1983; 56: 54-60.

21. Curi MM, Dib LL. Pinto DS. Management of solid ameloblastoma of the jaws with liquid nitrogen spray cryosurgery. Oral Surg Oral Med Oral Pathol Oral Radiol Endod 1997; 84: 339-44.
22. Kornblith AB, Zlotolow IM, Gooen J, Lerner T, Strong EW, Shah JP, Spiro RH, Holland JC. Quality of life of maxillectomy patients using an obturator prosthesis. Head Neck. 1996; 18(4): 323-34.

23. King GE, Martin JW. Complete dentures for obturator patient. Dent Clin North Am. 1996; 40 (1): 217-37.

24. Stephen A. Sachs: Sugical excision with peripheral ostectomy: a definitive, yet conservative, approach to the surgical management of ameloblastoma. J. Oral. Maxillofac. Surg. 2006; 64: 476-83.

25. Carlson ER, Marx RE: The ameloblastoma: primary, curative surgical management. J. Oral. Maxillofac. Surg.2006; 64: 484-94.

\section{CORRESPONDENCIA}

Dott. F. Carini

Ricercatore dell'Università degli Studi di MilanoBicocca.

Dipartimento di Odontostomatologia

Clinica Odontoiatrica: Ospedale San Gerardo Nuovo,

Villa Serena

Via Pergolesi, 33

20052 Monza (Mi)

E-mail: f.carini@hsgerardo.org fabrizio.carini@unimib.it 